\title{
Association of Nail Psoriasis With Disease Activity Measures and Impact in Psoriatic Arthritis: Data From the Corrona Psoriatic Arthritis/Spondyloarthritis Registry
}

\author{
Philip J. Mease ${ }^{1}$, Mei Liu², Sabrina Rebello², Robert R. McLean², Blessing Dube², Meghan Glynn², \\ Peter $\mathrm{Hur}^{3}$, and Alexis Ogdie ${ }^{4}$
}

\begin{abstract}
Objective. To examine the association of nail psoriasis with disease activity, quality of life, and work productivity in patients with psoriatic arthritis (PsA).

Methods. All patients with PsA who enrolled in the Corrona PsA/Spondyloarthritis Registry between March 2013 and October 2018 and had data on physician-reported nail psoriasis were included and stratified by presence vs absence of nail psoriasis at enrollment. Patient demographics, disease activity, quality of life (QOL), and work productivity at enrollment were compared between patients with vs without nail psoriasis using $t$-tests or Wilcoxon rank-sum tests for continuous variables and chi-square or Fisher exact tests for categorical variables.

Results. Of the 2841 patients with PsA included, 1152 (40.5\%) had nail psoriasis and 1689 (59.5\%) did not. Higher proportions of patients with nail psoriasis were male ( $51.9 \%$ vs $44.1 \%)$ and disabled from working ( $12.3 \%$ vs $7.8 \%$ ) compared with patients without nail psoriasis (all $P<0.05$ ). Patients with nail psoriasis had higher disease activity than those without nail psoriasis, including higher tender and swollen joint counts, worse Disease Activity Index for Psoriatic Arthritis and Psoriatic Arthritis Disease Activity Score values, and increased likelihood of having enthesitis and dactylitis (all $P<0.05)$. Patients with nail psoriasis had worse pain, fatigue, and work and activity impairment than those without nail psoriasis (all $P<0.05$ ).

Conclusion. Patients with PsA who have nail psoriasis had worse disease activity, QOL, and work productivity than those without nail involvement, emphasizing the importance of identification and management of nail disease in patients with PsA.
\end{abstract}

Key Indexing Terms: nail disease, psoriasis, psoriatic arthritis, registry

Psoriatic arthritis (PsA) is a chronic, immune-mediated inflammatory disease with a diverse array of symptoms that affect the musculoskeletal system, skin, and/or nails ${ }^{1}$. The Group for Research and Assessment of Psoriasis and Psoriatic Arthritis (GRAPPA) recognizes 6 clinical domains of PsA: peripheral disease, axial disease, enthesitis, dactylitis, skin disease, and nail disease ${ }^{2}$. Symptoms in these domains may occur alone or

This study was sponsored by Corrona, LLC. Corrona, LLC, has been supported through contracted subscriptions in the last 2 years by AbbVie, Amgen, Boehringer Ingelheim, Bristol Myers Squibb, Celgene, Crescendo, Eli Lilly and Company, Genentech, Gilead, GSK, Janssen, Merck,

Momenta Pharmaceuticals, Novartis, Ortho Dermatologics, Pfizer Inc., Regeneron, Roche, Sun, and UCB. The design and conduct of the study were a collaborative effort between Corrona, LLC, and Novartis, and financial support for the study was provided by Novartis. Novartis participated in the interpretation of data and review and approval of the manuscript.

${ }^{1}$ P.J. Mease, MD, Swedish Medical Center/Providence St. Joseph Health and University of Washington, Seattle, Washington; ${ }^{2} M$. Liu, PhD, S. Rebello, MPH, R.R. McLean, DSc, MPH, B. Dube, MPH, M. Glynn, MS, CPH, Corrona, LLC, Waltham, Massachusetts; ${ }^{3}$ P. Hur, PharmD, MBA, Novartis Pharmaceuticals Corporation, East Hanover, New Jersey; ${ }^{4} A$. Ogdie, MD, MCSE, Perelman School of Medicine at the University of Pennsylvania, Philadelphia, Pennsylvania, USA.

PJM has received research grants from Celgene, Novartis, AbbVie, Amgen, in combination and range from mild to severe 2 . In addition to clinical signs and symptoms, patients with PsA can experience reduced health-related QOL (HRQOL) from the pain, stiffness, fatigue, and reduced physical function that result from the disease ${ }^{3}$.

Nail disease is an important feature of PsA, and prevalence estimates of nail disease among patients with PsA range from

Bristol Myers Squibb, Eli Lilly, Pfizer, and UCB; consulting fees from Celgene, Corrona, Novartis, AbbVie, Amgen, Bristol Myers Squibb, Galapagos, Gilead, Janssen, Lilly, Merck, Pfizer, Sun, and UCB; and speakers bureau fees from AbbVie, Amgen, Bristol Myers Squibb, Celgene, Genentech, Janssen, Pfizer, and UCB. ML, SR, RRM, BD, and MG are employees of Corrona, LLC. PH is an employee of Novartis Pharmaceuticals Corporation. AO has received consulting fees from Amgen, AbbVie, Bristol Myers Squibb, Celgene, Lilly, Novartis, and Pfizer, and has received grant support from the National Institutes of Health/National Institute of Arthritis and Musculoskeletal and Skin Diseases, National Psoriasis Foundation, Rheumatology Research Foundation, Pfizer, and Novartis.

Address correspondence to Dr. P. Mease, Seattle Rheumatology Associates, 601 Broadway, Suite 600, Seattle, WA 98122, USA. Email:pmease@philipmease.com.

Full Release Article. For details see Reprints and Permissions at jrheum.org. Accepted for publication Oct 6, 2020. 
$41 \%$ to $93 \%{ }^{4}$. Manifestations of nail psoriasis differ based on the involved structure within the nail unit. Psoriasis in the nail matrix can present as pitting, crumbling, leukonychia, red spots of the lunula, and transverse grooves, whereas psoriasis in the nail bed may present as oil-drop discoloration, splinter hemorrhages, subungual hyperkeratosis, or onycholysis ${ }^{5,6}$. Additionally, imaging studies have indicated a close link between nail psoriasis and enthesitis (inflammation of the tendons, ligaments, or joint capsule fiber insertion into the bone $)^{7,8}$, a hallmark manifestation of early PsA that can lead to structural changes, pain, and disability?.

Patients with psoriatic disease who have nail involvement have worse HRQOL than those without nail involvement ${ }^{10,11}$. Nail psoriasis can result in pain and substantial functional impairment, which may inhibit daily activities and reduce work productivity ${ }^{11,12,13,14}$. Nail psoriasis is also associated with an increased prevalence of anxiety and depression, as well as psychological distress due to embarrassment, self-consciousness, and feeling stigmatized by what is perceived as a disfiguring disease $e^{6,10,15}$.

Although nail disease is an important feature of PsA, little is known about how patients with PsA and nail disease differ from those without nail disease with respect to patient and clinical characteristics and disease burden. A better understanding of the burden of nail disease in patients with PsA may help raise awareness of the importance of assessing nail involvement in patients with PsA and affect treatment choices. In patients with psoriasis (without PsA), nail involvement is associated with higher psoriasis disease severity, including a higher percentage of affected body surface area (BSA) and worse Psoriasis Area and Severity Index (PASI) scores ${ }^{11,16,17,18}$, more pain and psychological distress, reduced HRQOL, and substantial functional impairment and disability ${ }^{11,13,15,17,18}$. However, limited data are available regarding the burden of nail disease in PsA. Few real-world studies have examined characteristics of patients with PsA and nail psoriasis, particularly in the United States. The objective of this study was to examine the association of nail disease with patient demographics, disease activity, QOL, and work productivity of patients with PsA. We addressed this question using real-world data from the US-based Corrona PsA/Spondyloarthritis (SpA) Registry.

\section{MATERIALS AND METHODS}

Study design. This was a cross-sectional study conducted using data collected at the time of patient enrollment in the Corrona PsA/SpA registry.

Data source. The Corrona PsA/SpA Registry is a large, independent, prospective, observational cohort initiated in March 2013 that comprises patients diagnosed with PsA or SpA by a rheumatologist. The registry includes patients recruited by 49 participating rheumatologists from 45 private and academic practice sites across 27 states in the USA. As of October 1, 2018, data on approximately 3572 patients with PsA/SpA had been collected.

All participating investigators were required to obtain full board approval for conducting noninterventional research with a limited data set involving human participants. The Corrona PsA/SpA Registry and its investigators have been reviewed and approved by a central institutional review board (IRB; New England Independent Review Board No. 120160070). For academic investigative sites that did not receive a waiver to use the central IRB, full board approval was obtained from the respective governing IRB. All research was conducted in compliance with the current (2013) version of the Declaration of Helsinki. All registry participants were required to provide written informed consent and authorization prior to participating.

Study population. This study included all patients aged $\geq 18$ years enrolled in the Corrona PsA/SpA Registry between March 2013 and October 2018 with a diagnosis of PsA and nonmissing data on physician-reported nail psoriasis at the enrollment visit.

Exposure. Patients were stratified by presencevs absence of physician-reported nail psoriasis at the time of enrollment. Presence of nail psoriasis was defined as a nonzero response on the nail psoriasis visual analog scale (VAS) of $0-100$. The physician nail psoriasis VAS has been used in previous studies of patients with PsA and shown to correlate well with both the modified Nail Psoriasis Severity Index (mNAPSI) and patient-reported nail VAS scores ${ }^{19}$. No disease severity cutoffs have been established for the nail psoriasis VAS and we observed substantial variability in the distribution of nail psoriasis VAS scores in our study population. The lack of clear cutoffs precluded meaningful analysis of the correlation between the severity of nail involvement and disease activity measures. Thus, for the purposes of these analyses, patients were classified as those with nail psoriasis (nail psoriasis VAS score $\geq 1$ ) and those without nail psoriasis (nail psoriasis VAS score $=0$ ).

Study assessments. Data were collected at registry enrollment using questionnaires completed during office visits by patients and their treating rheumatologists. Data collected included patient demographics (age, sex, race, insurance type, and work status), clinical characteristics (BMI, symptom duration, disease duration, history of physician-reported comorbidities, treatment history, and current treatment), laboratory measurements (C-reactive protein and erythrocyte sedimentation rate), disease activity measures, and patient-reported outcome measures (PROM).

Disease activity measures evaluated in this study included nail psoriasis VAS, 68 tender joint count (TJC), 66 swollen joint count (SJC), Disease Activity Index for Psoriatic Arthritis (DAPSA) score, Psoriatic Arthritis Disease Activity Score (PASDAS), enthesitis, dactylitis, percentage of affected BSA, physician global assessment (PGA) of arthritis (VAS, 0-100), PGA of arthritis and psoriasis (VAS, $0-100$ ), and minimal disease activity (MDA). MDA was defined as "yes" if a patient met $\geq 5$ of the 7 following categories: $\mathrm{TJC} \leq 1, \mathrm{SJC} \leq 1, \mathrm{BSA} \leq 3 \%$, patient pain VAS $\leq 15$, patient global activity VAS $\leq 20$, Health Assessment Questionnaire-Disability Index $(\mathrm{HAQ}-\mathrm{DI}) \leq 0.5$, and tender entheseal points $\leq 1^{20}$. PROM included patient-reported pain (VAS, 0-100), patient-reported fatigue (VAS, 0-100), patient global assessment (PtGA) of arthritis (VAS, 0-100), PtGA of arthritis and psoriasis (VAS, 0-100), morning stiffness, HAQ-DI (0-3), EuroQol 5-dimension questionnaire (EQ-5D; 0-1), and EQ VAS (0-100). Work productivity was assessed using the Work Productivity and Activity Impairment Questionnaire.

Statistical analysis. Descriptive analyses of patient demographics, disease activity, QOL, and work productivity were assessed at enrollment. Categorical variables were summarized using frequency counts and percentages. Continuous variables were summarized using means and SD. Patients with and without nail psoriasis were compared using $t$-tests or Wilcoxon rank-sum tests for continuous variables and chi-square or Fisher exact tests for categorical variables. Statistical analyses were performed using Stata 15.1 (StataCorp).

\section{RESULTS}

Demographics, clinical characteristics, and treatment profile. Of the 2925 patients with PsA enrolled in the Corrona PsA/SpA Registry between March 2013 and October 2018, information was available for 2841 about the presence of nail psoriasis at registry enrollment and were included in the analysis. Of the included patients, 1152 had nail psoriasis (prevalence, 40.5\%). 
Overall, the mean age was 53.9 years, $52.7 \%$ of patients were female, most patients were White (94.0\%), were overweight or obese (83.7\%), and had a history of psoriasis (86.5\%; Table 1$)$.

The mean symptom and disease durations were 11.3 and 8.0

Table 1. Demographics, clinical characteristics, and treatment profile of patients with PsA at enrollment, stratified by presence of nail psoriasis.

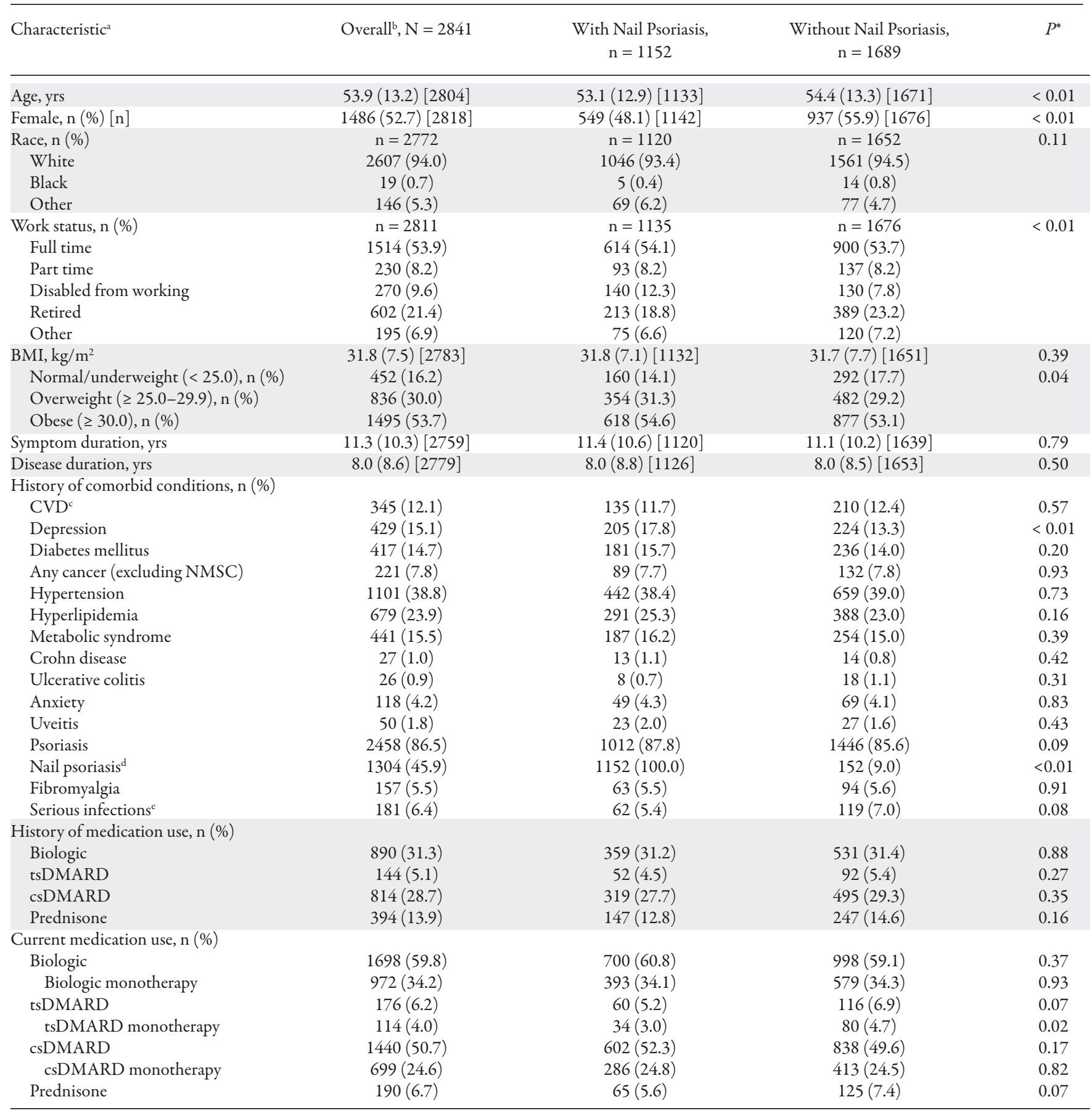

${ }^{a}$ All values were calculated based on available data and are presented as mean (SD) [n] unless otherwise stated. ${ }^{b}$ Patients with nonmissing nail VAS. ${ }^{\mathrm{c}} \mathrm{CVD}$ includes combined histories of myocardial infarction, acute coronary syndrome, coronary artery disease, congestive heart failure, peripheral arterial thromboembolic event, peripheral artery disease, cardiac revascularization procedure, ventricular arrhythmia, cardiac arrest, unstable angina, stroke, transient ischemic attack, peripheral ischemia or gangrene (necrosis), pulmonary embolism, carotid artery disease, or other cardiovascular event. ${ }^{\mathrm{d}}$ Determined by physician-reported nail psoriasis VAS $>0$ or clinical changes associated with nail changes or typical psoriatic nail dystrophy (may include the history of nail changes or typical psoriatic nail dystrophy prior to enrollment). ${ }^{e}$ Includes infections that led to hospitalization or intravenous antibiotics: joint/bursa, cellulitis, sinusitis, diverticulitis, sepsis, pneumonia, bronchitis, gastroenteritis, meningitis, urinary tract infection, upper respiratory tract infection, mycobacterium tuberculosis, or infection of other specified site. "Wilcoxon rank-sum test or $t$-test for continuous variables and chi-square or Fisher exact test for categorical variables. csDMARD: conventional synthetic disease-modifying antirheumatic drug; CVD: cardiovascular disease; NMSC: nonmelanoma skin cancer; PsA: psoriatic arthritis: tsDMARD: targeted synthetic antirheumatic drug; VAS: visual analog scale. 
years, respectively. Prior to registry enrollment, the majority of patients (68.7\%) were biologic-naïve. At the time of registry enrollment, $59.8 \%$ of patients were receiving a biologic.

Patients with nail psoriasis were slightly younger (mean age, 53.1 vs 54.4 yrs) and more likely to be male ( $51.9 \%$ vs $44.1 \%)$ compared with patients without nail psoriasis (both $P<0.01$; Table 1). Higher proportions of patients with nail psoriasis were disabled from working $(12.3 \%$ vs $7.8 \% ; P<0.01$ for distribution across work status category) and had a history of depression (17.8\% vs $13.3 \% ; P<0.01)$ compared with patients without nail psoriasis. Symptom duration, disease duration, and prior and current medication use were comparable in patients with vs without nail psoriasis.

Disease activity and PROM. Overall, patients with nail psoriasis had higher disease activity at enrollment than those without nail psoriasis (Table 2). Patients with nail psoriasis had a significantly higher mean percentage of affected BSA (7.9\% vs 3.5\%), TJC
(6.0 vs 3.5), and SJC (2.4 vs 1.7) and worse mean DAPSA (18.1 vs 13.4) and PASDAS (3.8 vs 3.3) than patients without nail psoriasis (all $P<0.01$ ). Patients with nail disease were less likely to have MDA (35.0\% vs $47.6 \%)$ and had an increased prevalence of enthesitis $(27.7 \%$ vs $17.0 \%)$ and dactylitis $(12.2 \%$ vs 7.4\%) compared with those without nail psoriasis (all $P<0.01$ ). Additionally, patients with nail psoriasis had higher mean PGA of arthritis (22.7 vs 17.0) and PGA of arthritis and psoriasis (27.2 vs 18.8 ) scores than those without nail psoriasis (both $P<0.01$ ).

Patients with nail psoriasis also had overall worse physical function and HRQOL than patients without nail psoriasis (Table 3). Patients with nail psoriasis reported higher mean pain (41.7 vs 36.1) and fatigue (43.9 vs 39.0) and had higher scores on their PtGA of arthritis (41.8 vs 38.0), and PtGA of arthritis and psoriasis (41.6 vs 37.4) than those without nail psoriasis (all $P<0.01)$. In addition, patients with nail psoriasis had worse mean HAQ-DI (0.7 vs 0.6$)$ and EQ VAS (68.6 vs 72.1) scores

Table 2. Disease activity among patients with PsA at enrollment, stratified by presence of nail psoriasis.

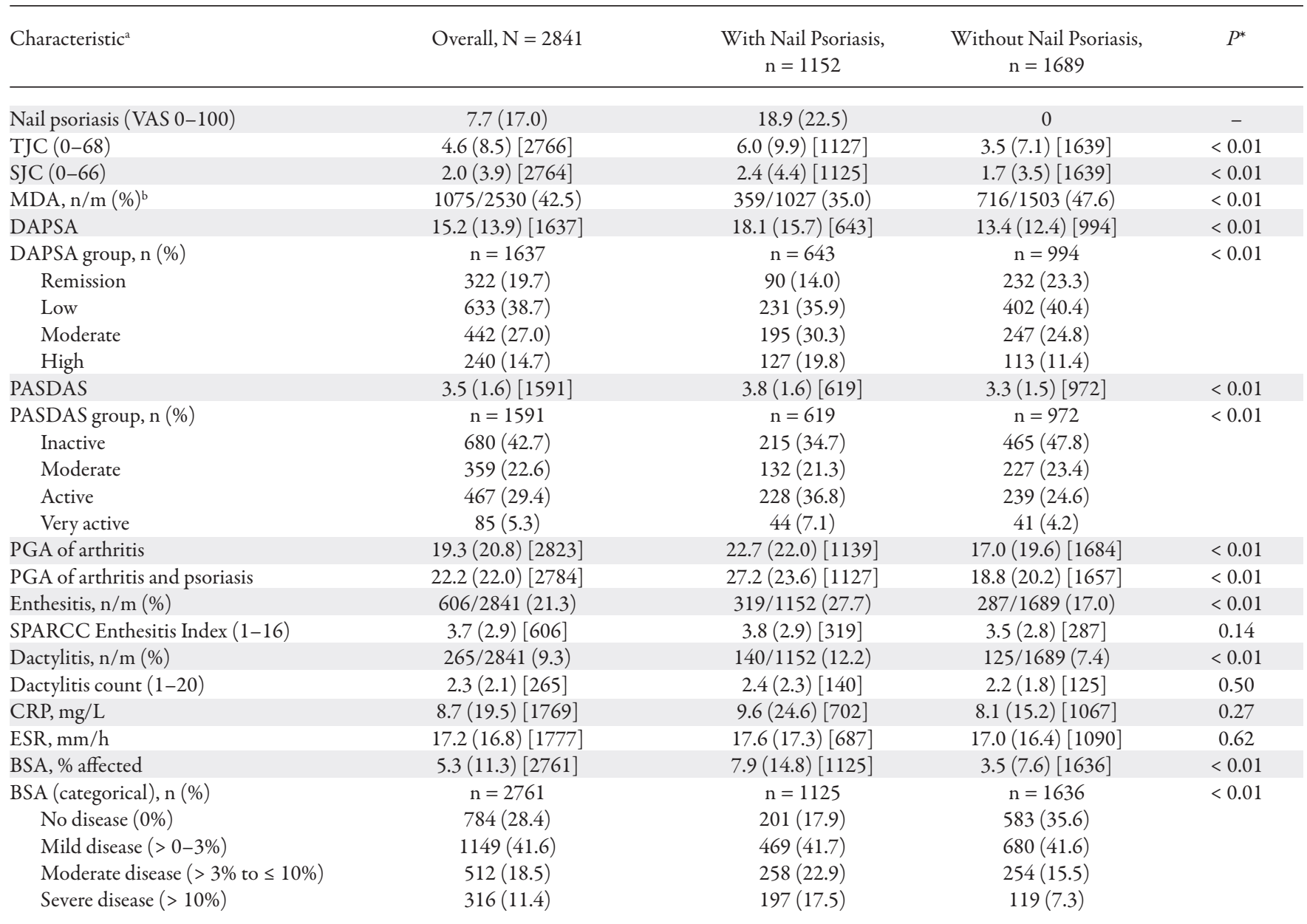

${ }^{a}$ All values were calculated based on available data and are presented as mean (SD) [n] unless otherwise stated. ${ }^{\mathrm{b}}$ MDA is defined as "yes" if a patient met $\geq 5$ of the 7 following categories: TJC $\leq 1$, SJC $\leq 1$, BSA $\leq 3 \%$, patient pain VAS $\leq 15$, patient global activity VAS $\leq 20$, HAQ-DI $\leq 0.5$, and tender entheseal points $\leq 1 .{ }^{*} t$-test or Wilcoxon rank-sum test for continuous variables and chi-square or Fisher exact test for categorical variables. BSA: body surface area; CRP: C-reactive protein; DAPSA: Disease Activity Index for Psoriatic Arthritis; ESR: erythrocyte sedimentation rate; HAQ-DI: Health Assessment Questionnaire-Disability Index; MDA: minimal disease activity; n/m: number of responders/number of patients with sufficient data for evaluation; PASDAS: Psoriatic Arthritis Disease Activity Score; PGA: physician global assessment; PsA: psoriatic arthritis; SJC: swollen joint count; SPARCC: Spondyloarthritis Research Consortium of Canada; TJC: tender joint count; VAS: visual analog scale. 
Table 3. Patient-reported outcome measures among patients with PsA at enrollment, stratified by presence of nail psoriasis.

\begin{tabular}{|c|c|c|c|c|}
\hline Characteristic $^{a}$ & Overall, $\mathrm{N}=2841$ & $\begin{array}{l}\text { With Nail Psoriasis, } \\
\qquad \mathrm{n}=1152\end{array}$ & $\begin{array}{l}\text { Without Nail Psoriasis, } \\
\qquad \mathrm{n}=1689\end{array}$ & $P^{*}$ \\
\hline Patient-reported pain (VAS 0-100) & $38.4(29.6)[2684]$ & $41.7(30.1)[1081]$ & $36.1(29.1)[1603]$ & $<0.01$ \\
\hline PtGA of arthritis (VAS 0-100) & $39.6(29.9)[2795]$ & $41.8(29.5)[1132]$ & $38.0(30.1)[1663]$ & $<0.01$ \\
\hline PtGA of arthritis and psoriasis (VAS 0-100) & $39.1(29.7)[2789]$ & $41.6(29.0)[1130]$ & $37.4(30.0)[1659]$ & $<0.01$ \\
\hline Morning stiffness & $\mathrm{n}=2762$ & $\mathrm{n}=1118$ & $\mathrm{n}=1644$ & 0.1 \\
\hline$\geq 30 \mathrm{~min}$ & $1782(64.5)$ & $738(66.0)$ & $1044(63.5)$ & \\
\hline HAQ-DI (0-3) & $0.7(0.6)[2700]$ & $0.7(0.7)[1087]$ & $0.6(0.6)[1613]$ & $<0.01$ \\
\hline EQ-5D $(0-1)$ & $0.8(0.2)[2680]$ & $0.7(0.2)[1080]$ & $0.8(0.2)[1600]$ & $<0.01$ \\
\hline EQ VAS $(0-100)$ & $70.7(21.3)[2790]$ & $68.6(21.4)[1127]$ & $72.1(21.1)[1663]$ & $<0.01$ \\
\hline \multicolumn{5}{|l|}{ WPAI domains } \\
\hline Current employment, n/m (\%) & $1681 / 2745(61.2)$ & $678 / 1109(61.1)$ & $1003 / 1636(61.3)$ & 0.93 \\
\hline
\end{tabular}

${ }^{a}$ All values were calculated based on available data and are presented as mean (SD) [n] unless otherwise indicated. ${ }^{*}$ Wilcoxon rank-sum test $t$-test or for continuous variables and chi-square or Fisher exact test for categorical variables. EQ-5D: EuroQol 5-dimension questionnaire; HAQ-DI: Health Assessment Questionnaire-Disability Index; MDA: minimal disease activity; $\mathrm{n} / \mathrm{m}$ : number of responders/number of patients with sufficient data for evaluation; PsA: psoriatic arthritis; PtGA: patient global assessment; VAS: visual analog scale; WPAI: Work Productivity and Activity Impairment Questionnaire.

than patients without nail psoriasis (both $P<0.01$ ). Patients with nail psoriasis also reported greater mean percentages of impairment while working $(20.3 \%$ vs $18.1 \% ; P=0.02)$, overall work impairment $(23.2 \%$ vs $20.5 \% ; P=0.02)$, and activity impairment $(32.5 \%$ vs $26.6 \% ; P<0.01)$ than those without nail psoriasis.

\section{DISCUSSION}

Although nail psoriasis is recognized as a common feature of PsA, few studies have examined the relevance of nail disease for treatment and outcomes in PsA. Whereas a handful of studies have quantified the prevalence of nail psoriasis in PsA, to our knowledge, our study is among the first to evaluate the burden of nail disease in a real-world population of patients with PsA. We found that patients with PsA in our study who had nail psoriasis had a higher percentage of affected BSA and higher PsA disease activity, including higher TJC, SJC, PASDAS, and DAPSA scores, than those without nail psoriasis. Additionally, the prevalence of work disability and depression was higher among patients with PsA with nail psoriasis, and patients with nail psoriasis had worse pain, fatigue, and EQ VAS scores, and more work and activity impairment than those without nail psoriasis. Our results show that nail psoriasis was associated with an overall higher burden of disease in all domains of PsA.

Nail psoriasis is closely linked with enthesitis, an inflammatory change that occurs early in the development of $\mathrm{PsA}^{8,21}$. This relationship may be due in part to the close anatomical association of the nail matrix with the extensor tendon of the distal interphalangeal joint, an entheseal site frequently affected in early $\mathrm{PsA}^{7,8,9,22}$. In our study population, a higher proportion of patients with nail psoriasis had enthesitis compared with those without nail involvement, which may be reflective of the relationship between nail psoriasis and enthesitis. In patients with PsA, enthesitis is associated with more peripheral and axial joint damage, higher disease activity, worse HRQOL, and more functional impairment ${ }^{23,24}$. Thus, when selecting therapies for a patient with PsA with nail involvement, it may be beneficial to consider therapies that can effectively treat nail disease. Available treatments for nail psoriasis include topical therapies, corticosteroid injections, conventional synthetic disease-modifying antirheumatic drugs (csDMARD), and biologics ${ }^{5,6,25,26,27}$. Current guidelines recommend that choice of therapy should take into account the presence of symptoms in other PsA domains, such as severe skin involvement, presence of enthesitis or dactylitis, and presence of inflammatory bowel disease, as well as patient factors such as medication history and preferences for mode of administration and frequency of dosing $2,28,29,30,31,32$.

In our real-world population of US patients with PsA, 40.5\% of patients had nail psoriasis at the time of registry enrollment. The prevalence of nail involvement in our study population is lower than that observed in previous real-world studies of patients with varied prior biologic experience, which have reported rates of nail involvement ranging from 50\% to $87 \%$ among patients with $\mathrm{PsA}^{33-39}$. Notably, approximately $31 \%$ of patients in our study population had received biologic therapy and $29 \%$ had received csDMARD prior to enrollment, which may have ameliorated nail disease in those patients before their participation in the study ${ }^{5,26}$. Additionally, the majority of previous studies specifically enrolled patients with PsA and psoriasis, whereas the presence of psoriasis was not a criterion for enrollment in our study.

This study is subject to the general limitations of real-world observational studies. Although patients enrolled in the Corrona PsA/SpA Registry are assumed to be generally representative of 
patients with PsA in the USA, there may be differences in generalizability overall, given that most patients are enrolled in the registry at the time of therapy initiation ${ }^{40}$. All comparisons were descriptive; no adjustments were made to account for differences in patient characteristics, such as age and sex, which may have influenced the other differences observed in patients with vs without nail psoriasis. In this cross-sectional study, adjusting for some of the disease activity elements at baseline was colinear with the outcome of interest and was therefore considered inappropriate for this analysis. Future cohort studies that examine the implications of nail disease on disease activity outcomes should address the effect of higher baseline disease activity. The presence of nail psoriasis was based on physician reporting and may have been underreported. Information on duration and subtypes (e.g., pitting, onycholysis) of nail psoriasis was not captured. The overall severity of nail involvement was evaluated on a VAS, but the link between VAS severity and disease burden was not specifically assessed, as cutoffs have not been established for this measure.

Overall, the results of this study highlight the burden of nail psoriasis in patients with PsA. Nail psoriasis was associated with greater severity of psoriasis and PsA symptoms, more disability and functional impairment, and worse HRQOL. These findings emphasize the importance of identification and management of nail disease in patients with PsA. Further research is needed to assess whether nail disease affects treatment response in patients with PsA.

\section{ACKNOWLEDGMENTS}

The authors thank the participating providers and patients for contributing data to the Corrona PsA/SpA Registry. Support for third-party writing assistance for this manuscript, furnished by Elizabeth Ohneck, PhD, of Health Interactions Inc, was provided by Novartis Pharmaceuticals Corporation, East Hanover, New Jersey, USA.

\section{REFERENCES}

1. Gladman DD. Clinical features and diagnostic considerations in psoriatic arthritis. Rheum Dis Clin North Am 2015;41:569-79.

2. Coates LC, Kavanaugh A, Mease PJ, Soriano ER, Acosta-Felquer ML, Armstrong AW, et al. Group for Research and Assessment of Psoriasis and Psoriatic Arthritis 2015 treatment recommendations for psoriatic arthritis. Arthritis Rheumatol 2016;68:1060-71.

3. Gudu T, Gossec L. Quality of life in psoriatic arthritis. Expert Rev Clin Immunol 2018;14:405-17.

4. Ogdie A, Weiss P. The epidemiology of psoriatic arthritis. Rheum Dis Clin North Am 2015;41:545-68.

5. Pasch MC. Nail psoriasis: a review of treatment options. Drugs 2016;76:675-705.

6. Sobolewski P, Walecka I, Dopytalska K. Nail involvement in psoriatic arthritis. Reumatologia 2017;55:131-5.

7. Tan AL, McGonagle D. Psoriatic arthritis: correlation between imaging and pathology. Joint Bone Spine 2010;77:206-11.

8. McGonagle D. Enthesitis: an autoinflammatory lesion linking nail and joint involvement in psoriatic disease. J Eur Acad Dermatol Venereol 2009;23 Suppl 1:9-13.

9. Kaeley GS, Eder L, Aydin SZ, Gutierrez M, Bakewell C. Enthesitis: a hallmark of psoriatic arthritis. Semin Arthritis Rheum 2018; 48:35-43.
10. Klaassen KM, van de Kerkhof PC, Pasch MC. Nail psoriasis, the unknown burden of disease. J Eur Acad Dermatol Venereol 2014;28:1690-5.

11. Callis Duffin K, Mason MA, Gordon K, Harrison RW, Crabtree MM, Guana A, et al. Characterization of patients with psoriasis in challenging-to-treat body areas in the Corrona Psoriasis Registry. Dermatology 2020:1-10.

12. Jiaravuthisan MM, Sasseville D, Vender RB, Murphy F, Muhn CY. Psoriasis of the nail: anatomy, pathology, clinical presentation, and a review of the literature on therapy. J Am Acad Dermatol 2007; 57:1-27.

13. Baran R. The burden of nail psoriasis: an introduction. Dermatology 2010;221 Suppl 1:1-5.

14. Langley RG, Saurat JH, Reich K, Nail Psoriasis Delphi Expert Panel. Recommendations for the treatment of nail psoriasis in patients with moderate to severe psoriasis: a dermatology expert group consensus. J Eur Acad Dermatol Venereol 2012;26:373-81.

15. de Jong EM, Seegers BA, Gulinck MK, Boezeman JB, van de Kerkhof PC. Psoriasis of the nails associated with disability in a large number of patients: results of a recent interview with 1,728 patients. Dermatology 1996;193:300-3.

16. Armesto S, Esteve A, Coto-Segura P, Drake M, Galache C, Martínez-Borra J, et al. [Nail psoriasis in individuals with psoriasis vulgaris: a study of 661 patients]. [Article in Spanish] Actas Dermosifiliogr 2011;102:365-72.

17. Augustin M, Reich K, Blome C, Schäfer I, Laass A, Radtke MA. Nail psoriasis in Germany: epidemiology and burden of disease. Br J Dermatol 2010;163:580-5.

18. Radtke MA, Langenbruch AK, Schäfer I, Herberger K, Reich K, Augustin M. Nail psoriasis as a severity indicator: results from the PsoReal study. Patient Relat Outcome Meas 2011;2:1-6.

19. Cassell SE, Bieber JD, Rich P, Tutuncu ZN, Lee SJ, Kalunian KC, et al. The modified Nail Psoriasis Severity Index: validation of an instrument to assess psoriatic nail involvement in patients with psoriatic arthritis. J Rheumatol 2007;34:123-9.

20. Coates LC, Cook R, Lee KA, Chandran V, Gladman DD. Frequency, predictors, and prognosis of sustained minimal disease activity in an observational psoriatic arthritis cohort. Arthritis Care Res 2010;62:970-6.

21. Raposo I, Torres T. [Nail psoriasis as a predictor of the development of psoriatic arthritis]. [Article in Spanish] Actas Dermosifiliogr 2015;106:452-7.

22. Radtke MA, Beikert FC, Augustin M. Nail psoriasis - a treatment challenge. J Dtsch Dermatol Ges 2013;11:203-19; quiz 220.

23. Polachek A, Cook R, Chandran V, Gladman DD, Eder L. The association between sonographic enthesitis and radiographic damage in psoriatic arthritis. Arthritis Res Ther 2017;19:189.

24. Mease PJ, Karki C, Palmer JB, Etzel CJ, Kavanaugh A, Ritchlin CT, et al. Clinical characteristics, disease activity, and patient-reported outcomes in psoriatic arthritis patients with dactylitis or enthesitis: results from the Corrona Psoriatic Arthritis/Spondyloarthritis Registry. Arthritis Care Res 2017;69:1692-9.

25. Armstrong AW, Tuong W, Love TJ, Carneiro S, Grynszpan R, Lee SS, et al. Treatments for nail psoriasis: a systematic review by the GRAPPA Nail Psoriasis Work Group. J Rheumatol 2014; 41:2306-14

26. Haneke E. Nail psoriasis: clinical features, pathogenesis, differential diagnoses, and management. Psoriasis 2017;7:51-63.

27. Ventura A, Mazzeo M, Gaziano R, Galluzzo M, Bianchi L, Campione E. New insight into the pathogenesis of nail psoriasis and overview of treatment strategies. Drug Des Devel Ther 2017;11:2527-35.

28. Gossec L, Smolen JS, Ramiro S, de Wit M, Cutolo M, Dougados $\mathrm{M}$, et al. European League Against Rheumatism (EULAR) 
recommendations for the management of psoriatic arthritis with pharmacological therapies: 2015 update. Ann Rheum Dis 2016;75:499-510.

29. Singh JA, Guyatt G, Ogdie A, Gladman DD, Deal C, Deodhar A, et al. Special article: 2018 American College of Rheumatology/ National Psoriasis Foundation guideline for the treatment of psoriatic arthritis. Arthritis Care Res 2019;71:2-29.

30. Menter A, Strober BE, Kaplan DH, Kivelevitch D, Prater EF, Stoff $\mathrm{B}$, et al. Joint AAD-NPF guidelines of care for the management and treatment of psoriasis with biologics. J Am Acad Dermatol 2019;80:1029-72.

31. Crowley JJ, Weinberg JM, Wu JJ, Robertson AD, Van Voorhees AS, National Psoriasis Foundation. Treatment of nail psoriasis: best practice recommendations from the Medical Board of the National Psoriasis Foundation. JAMA Dermatol 2015;151:87-94.

32. Rigopoulos D, Baran R, Chiheb S, Daniel CR III, Di Chiacchio N, Gregoriou S, et al. Recommendations for the definition, evaluation, and treatment of nail psoriasis in adult patients with no or mild skin psoriasis: a dermatologist and nail expert group consensus. J Am Acad Dermatol 2019;81:228-40

33. Papadavid E, Katsimbri P, Kapniari I, Koumaki D, Karamparpa A, Dalamaga M, et al. Prevalence of psoriatic arthritis and its correlates among patients with psoriasis in Greece: results from a large retrospective study. J Eur Acad Dermatol Venereol 2016; 30:1749-52.
34. Yan D, Ahn R, Leslie S, Liao W. Clinical and genetic risk factors associated with psoriatic arthritis among patients with psoriasis. Dermatol Ther 2018;8:593-604.

35. Kumar R, Sharma A, Dogra S. Prevalence and clinical patterns of psoriatic arthritis in Indian patients with psoriasis. Indian J Dermatol Venereol Leprol 2014;80:15-23.

36. Truong B, Rich-Garg N, Ehst BD, Deodhar AA, Ku JH, Vakil-Gilani K, et al. Demographics, clinical disease characteristics, and quality of life in a large cohort of psoriasis patients with and without psoriatic arthritis. Clin Cosmet Investig Dermatol 2015;8:563-9.

37. Zenke Y, Ohara Y, Kobayashi D, Arai S, Kishimoto M, Okada M, et al. Nail findings in patients with psoriatic arthritis: a cross-sectional study with special reference to transverse grooves. J Am Acad Dermatol 2017;77:863-7.

38. Zargari O, Leyli EK, Azimi SZ. Nail involvement in patients with psoriatic arthritis in northern Iran. Autoimmune Dis 2018;2018:4608490.

39. Krajewska-Wlodarczyk M, Owczarczyk-Saczonek A, Placek W, Wojtkiewicz M, Wiktorowicz A, Wojtkiewicz J. Ultrasound assessment of changes in nails in psoriasis and psoriatic arthritis. Biomed Res Int 2018;2018:8251097.

40. Gladman DD, Farewell VT. Longitudinal cohort studies. J Rheumatol Suppl 2005;72:30-2. 